\title{
Francesco Vissani
}

\section{INFN, Laboratori Nazionali del Gran Sasso and Gran Sasso Science Institute, L'Aquila, Italy}

\section{SOLAR NEUTRINO PHYSICS ON THE BEGINNING OF 2017}

This writeup is a review of current hot topics on solar neutrinos. It is based on a talk at the conference "Neutrinos: the quest for a new physics scale", held at the CERN on March 2017, where the Organizers entrusted me with a discussion of the provocative question "whether solar neutrino physics is over". Rather than providing a straight (negative) answer, in view of an audience consisting mostly of colleagues working in theoretical particle physics, I deemed it more useful providing a description of what is the current activity of the physicists working in solar neutrinos, leaving the listener free of forming his/her own opinion apropos.

Keywords: solar neutrinos, neutrino oscillations, nuclear astrophysics, $p p$ chain, CNO cycle.

\section{Introduction}

The study of solar neutrinos rests on solid scientific foundations. The single most important book in this field is still the one of Bahcall [1], a pioneer and a renowned astrophysicist, but recall that Bahcall initially was a nuclear physicist. His book emphasizes, already from the title, the centrality of astrophysical considerations. Another equally influential book is the one of Raffelt [2], whose scientific attitude, also in this case declared from the title, is the one of particle physicists instead. The content and the points of view of these books are still valid and useful for the understanding of solar neutrinos.

The only important change since the appearance of these books is the proof of neutrino "oscillations", or more precisely of neutrino transformations, as observed by various solar neutrino telescopes and other experiments. Two years ago the Nobel prize committee certified the occurrence of this phenomenon by awarding the leaders of Super-Kamiokande and SNO experiments. Neutrino transformations are an accepted fact and the long discussion that went under the heading of "solar neutrino anomaly" can be considered closed. Now we can proceed to compare accurately observations and expectations on solar neutrinos, testing our understanding of astrophysics and learning more interesting things.

One year ago, an entire issue of Eur. Phys. J. A was devoted to solar neutrinos [3]; there, several updated works and useful review papers can be found.

\section{The terms of the discussion}

There is a great interest toward the particle physics aspects concerning the neutrinos. However, in order not to loose the correct perspective or even to undermine the chances of doing good science, it is essential to keep in mind that solar neutrinos are a typical branch of astroparticle physics. Their proper understanding involves many fields of science, not only theoretical particle physics but also nuclear physics, astrophysics, astronomy and also experimental nuclear/particle physics. As in any field, there are some jargonic terms of the solar neutrino field cherished by the tradition and often used, that however make less easy to appreciate the current debate. Therefore, we begin simply by discussing the acronyms / main terms / keywords that recur in the discussion, and in particular: SSM, ES, PP, $\mathrm{CNO} /$ helioseismology, metallicity, Boron neutrinos, Borexino.

Expectations. The nuclear physics processes by which the Sun produces energy are listed in Table 1; they can be grouped in a chain of reaction named PP chain and in the catalytic CNO cycle. An important locution is that of "Standard Solar Model" whose acronym is SSM. It is used either generically or to denote the model originally developed by J. Bahcall and improved by several collaborators. This was and it is a tool of essential importance for the investigations. It is the comparison of the SSM and of the measurements in Homestake, Kamiokande, GALLEX/GNO, SAGE, that proved the existence of physics beyond the standard model, see e.g., [5] and convinced the scientific community that the "solar neutrino anomaly" was worth investigating further. Even the formulation of the principle of the SNO detector [6] relied heavily on SSM, despite of the fact that the measurement of the neutral current events of SNO is often termed a "model independent test". The study of the oscillations of solar surface, connected to the understanding of the matter distribution in the outermost layers of the Sun, a discipline called helioseismology, has lead to overall and independent validation of the SSM. Of particular importance are the trace elements beyond hydrogen and helium, called collectively "heavier elements" or referred as metals in astrophysical parlance. The predicted inner structure of the Sun depends upon their abundance. The existing and supposedly precise measurements lead however to contradicting SSM predictions [7], a problem that to date is still unsolved. The most recent version of the SSM 
appeared just a few months ago [8]; this study assesses the missing information, quantifies the short- comings of the model and furthermore examines the possible key tests.

\section{Table 1. Nuclear reactions in the Sun, adapted from [1]}

\begin{tabular}{|c|c|c|c|}
\hline Name & Reaction & $Q$-value, $\mathrm{keV}$ & $E_{\mathrm{v}}^{\max }, \mathrm{keV}$ \\
\hline PPI & $\begin{array}{l}p+p \rightarrow \mathrm{D}+\beta^{+}+v_{e} \\
p+\mathrm{D} \rightarrow{ }^{3} \mathrm{He}+\gamma \\
{ }^{3} \mathrm{He}+{ }^{3} \mathrm{He} \rightarrow \alpha+2 p\end{array}$ & $\begin{array}{c}1442 \\
5494 \\
12860\end{array}$ & 420 \\
\hline pep & $p+p+e \rightarrow \mathrm{D}+v_{e}$ & 1442 & 1442 \\
\hline PP II & $\begin{array}{l}{ }^{3} \mathrm{He}+{ }^{4} \mathrm{He} \rightarrow{ }^{7} \mathrm{Be}+\gamma \\
{ }^{7} \mathrm{Be}+e \rightarrow{ }^{7} \mathrm{Li}+v_{e} \\
{ }^{7} \mathrm{Li}+p \rightarrow 2 \alpha\end{array}$ & $\begin{array}{c}1586 \\
862,384 \\
17347\end{array}$ & 862,384 \\
\hline PP III & $\begin{array}{l}{ }^{7} \mathrm{Be}+p \rightarrow{ }^{8} \mathrm{~B}+\gamma \\
{ }^{8} \mathrm{~B} \rightarrow{ }^{8} \mathrm{Be}^{*}+\beta^{+}+v_{e} \\
{ }^{8} \mathrm{Be}^{*} \rightarrow 2 \alpha\end{array}$ & $\begin{array}{c}137 \\
18471-E_{x} \\
E_{x}\end{array}$ & $14600 \div 15100$ \\
\hline hep (PP IV) & ${ }^{3} \mathrm{He}+p \rightarrow \alpha+\beta^{+}+v_{e}$ & 19795 & 18773 \\
\hline $\mathrm{CNO} \mathrm{I}$ & $\begin{array}{l}{ }^{12} \mathrm{C}+p \rightarrow{ }^{13} \mathrm{~N}+\gamma \\
{ }^{13} \mathrm{~N} \rightarrow{ }^{13} \mathrm{C}+\beta^{+}+v_{e} \\
{ }^{13} \mathrm{C}+p \rightarrow{ }^{14} \mathrm{~N}+\gamma \\
{ }^{14} \mathrm{~N}+p \rightarrow{ }^{15} \mathrm{O}+\gamma \\
{ }^{15} \mathrm{O} \rightarrow{ }^{15} \mathrm{~N}+\beta^{+}+v_{e} \\
{ }^{15} \mathrm{~N}+p \rightarrow{ }^{12} \mathrm{C}+\alpha\end{array}$ & $\begin{array}{l}1943 \\
2221 \\
7551 \\
7297 \\
2754 \\
4966\end{array}$ & 1199 \\
\hline
\end{tabular}

$\mathrm{N}$ o t e. The first 11 reactions are the PP chain, grouped in 5 branches; the last 6 are part of cold CNO cycle that contributes little to solar luminosity. The 2nd reaction of PP II branch is an electron capture and produces two neutrino lines; the 2nd reaction of the PP III branch depends on the energy of the excited ${ }^{8} \mathrm{Be}$ state $E_{x}$ that is not known precisely [4]. Particles or atomic nuclei are indicated; $p={ }^{1} \mathrm{H}$ and $\mathrm{D}={ }^{2} \mathrm{H}$. For final states the traditional Rutherford's notation $\alpha={ }^{4} \mathrm{He}$ and $\beta^{+}=e^{+}$ is used. The energy of the positron is included in $Q$.

Experiments. It is funny to note that there are just two solar neutrino experiments that have been realized mostly to study the functioning of the Sun: the first one, Homestake and the last one, Borexino.

In the meantime we had other excellent experiments, which contributed to the field but which were aimed, mostly, to understand neutrinos rather than the Sun as summarized in Table 2.

Table 2. Main characteristics of solar neutrino experiments, including the minimum neutrino energy that yields an observable signal

\begin{tabular}{|c|c|c|c|c|c|}
\hline $\begin{array}{l}\text { Experiment } \\
\text { name }\end{array}$ & $\begin{array}{l}\text { Experiment } \\
\text { type }\end{array}$ & \multicolumn{2}{|c|}{$\begin{array}{c}\text { Neutrino } \\
\text { detection reaction }\end{array}$} & $\begin{array}{c}\text { Energy } \\
\text { measured }\end{array}$ & $\begin{array}{c}\text { Minimum } \\
v \text { energy, } \mathrm{keV}\end{array}$ \\
\hline Homestake & radiochemical & $v_{e}+{ }^{37} \mathrm{Cl} \rightarrow e+{ }^{37} \mathrm{Ar}$ & (CC) & no & 814 \\
\hline Gallex/GNO & radiochemical & $v_{e}+{ }^{71} \mathrm{Ga} \rightarrow e+{ }^{71} \mathrm{Ge}$ & (CC) & no & 233 \\
\hline SAGE & radiochemical & $v_{e}+{ }^{71} \mathrm{Ga} \rightarrow e+{ }^{71} \mathrm{Ge}$ & (CC) & no & 233 \\
\hline SNO & heavy water & $v+\mathrm{D} \rightarrow v+p+n$ & $(\mathrm{NC})$ & no & 2230 \\
\hline SNO & heavy water & $v_{e}+\mathrm{D} \rightarrow p+p+e$ & (CC) & yes & 4940 \\
\hline Kamiokande & water Cherenkov & $v+e \rightarrow v+e$ & $(\mathrm{CC}+\mathrm{NC})$ & yes & 7750 \\
\hline Super-K & water Cherenkov & $v+e \rightarrow v+e$ & $(\mathrm{CC}+\mathrm{NC})$ & yes & 3730 \\
\hline Borexino & ultra-pure scintillator & $v+e \rightarrow v+e$ & $(\mathrm{CC}+\mathrm{NC})$ & yes & 285 \\
\hline
\end{tabular}

$\mathrm{N}$ o t e. The first 4 entries refer to experiments that count the nuclei or neutrons due to neutrino interactions. The last 4 entries instead describe experiments capable to measure the energy of the electrons in the final state with energies above 3.5, 7.5, 3.49 and $0.15 \mathrm{MeV}$, respectively. $\mathrm{CC}$ and $\mathrm{NC}$ are charged current and neutral current reaction, respectively.

There are many differences among these experiments. Homestake and the Gallium experiments observe all neutrino interactions above a certain energy threshold, using neutrino interactions on nuclei, whose cross sections are known less precise- ly than those used by the other experiments. Kamiokande and its successor Super-Kamiokande (often denoted as Super-K), SNO and Borexino, instead, have been able to observe the differential spectrum. Let us recall that Borexino, just as Kami- 
okande / Super-Kamiokande, detects solar neutrinos thanks to the neutrino elastic scattering on atomic electrons, ES for short, namely

$$
v+e \rightarrow v+e
$$

The cross section of ES is theoretically very clean; radiative corrections in the standard model are known and the precision is much better than what currently needed. However, in scintillator detectors such as Borexino, KamLAND or the future detector JUNO, the direction of the single electron cannot be reconstructed and background cannot be discriminated: for this reason, it is necessary to be sure a priori of the absence of radioactive contaminants. Borexino proved that a condition of ultrahigh radiopurity is achievable and moreover the light yield of electrons is high, which allows one to measure the energy precisely, with few \% precision, down to very low energies. This experiment succeeded to achieve an impressive energy threshold of about $150 \mathrm{keV} .{ }^{1}$

\section{Nuclear astrophysics}

The PP chain. In Fig. 1 we summarize the expectations for solar neutrinos as a differential spectrum, and show the range of energies explored by Super-K, SNO and Borexino, namely the solar neutrino experiments that are capable to measure the energy of the events and to separate the various neutrinos.

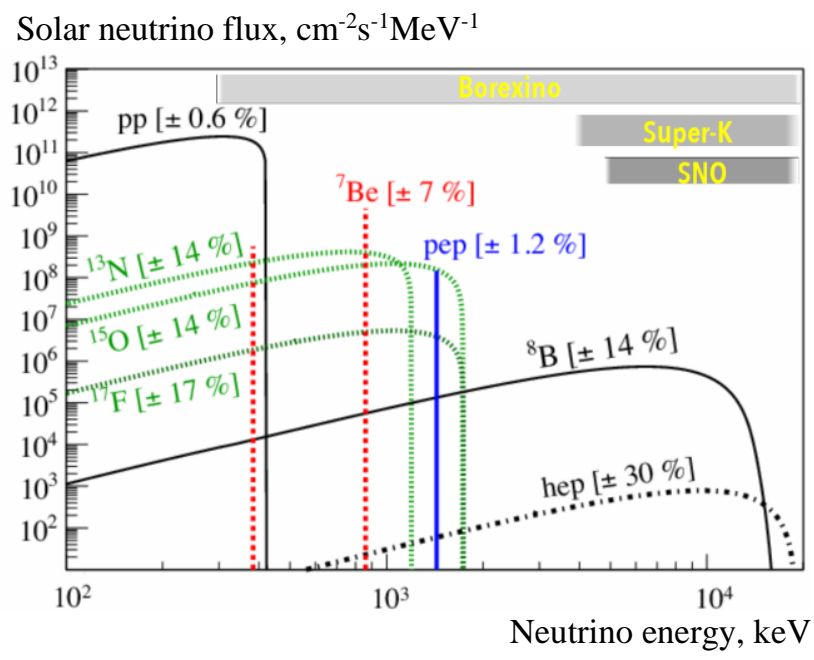

Fig. 1. The solar neutrino spectrum predicted by the SSM of Bahcall and collaborators, as compiled by [9]. The three grey strips indicate the energy ranges of operation of the solar neutrino telescopes able to measure the differential spectrum. Compare with Tables 1 and 2; see the text for discussion. (See color Figure on the journal website.)

${ }^{1}$ The minimum neutrino energy is when the final state neutrino recoils backward. Summing the energy and momentum conservation conditions $E_{\mathrm{v}}+m_{e}=E_{\mathrm{v}}{ }^{\prime}+E_{e}$ and $E_{v}=-E_{v}{ }^{\prime}+p_{e}$ we get $E_{v}{ }^{m i n}=\left(T_{e}+p_{e}\right) / 2$.
Let us begin by discussing the high energy solar neutrinos. Those produced in the PP III branch of the PP chain in Table 1, the so called Boron neutrinos, have been investigated accurately thanks to all these experiments. Super-K and SNO contributed mostly, since Borexino is by far smaller. The latter, however, probed Boron neutrinos till the lowest energies ever achieved and it is planning to go down till $2.5 \mathrm{MeV}$. The highest energy neutrinos, so called hep belonging to PP IV branch, are still unobserved to date owing to the small flux; their search is continued by Super-K and will be continued in future large detectors as Hyper-K and maybe JUNO.

However, the above neutrinos amount only to $0.02 \%$ of the neutrinos emitted by the Sun. All the other neutrinos belonging to the PP chain, namely, PP, pep, Beryllium, have been observed directly only by Borexino. A few remarks on PP neutrinos are in order. As evident from Fig. 1, these neutrinos are the most abundant among solar neutrinos and those directly related to the principal nuclear chain of energy generation in the Sun. Thus, it is possible to say that, after the observation of PP neutrinos, we have sound experimental bases to the basic understanding of how the Sun functions. It should be emphasized that this measurement is quite recent, having been obtained just 3 years ago [11]. Note that solar neutrinos flow out immediately after being produced, whereas the electromagnetic radiation does it several 100 thousands years later. Since this is a small time in comparison to its lifetime, it is possible (and easy) to relate the flux of PP neutrinos just to the solar luminosity observed today: this makes very reliable the theoretical expectation. Conversely, the observation of PP neutrinos ensures us that the Sun will continue to work as it is currently doing for several 100 thousand years at least. The SSM (i.e., the theory) predicts that the increased luminosity of the Sun will vaporize terrestrial oceans after one billion years; its life as a star will end after four billion years or so.

The CNO cycle. The CNO cycle depicted in Fig. 2 is the main cycle of energy generation in the most massive stars. This is expected to yield $\sim 1 \%$ of the solar luminosity and it is not explored yet. Its measurement may help us to fix the pending issues of SSM and moreover it is a unique occasion to check the reliability of our understanding of stellar

2 The name "Borexino" derives from the one of a previously proposed experiment, BOREX, that was supposed to be loaded with ${ }^{11} \mathrm{~B}$ and that was aimed at observing Boron neutrinos [10]. The concept of the experiment was radically modified but the connection with boron remained in the name and thanks to the results on Boron neutrinos. 


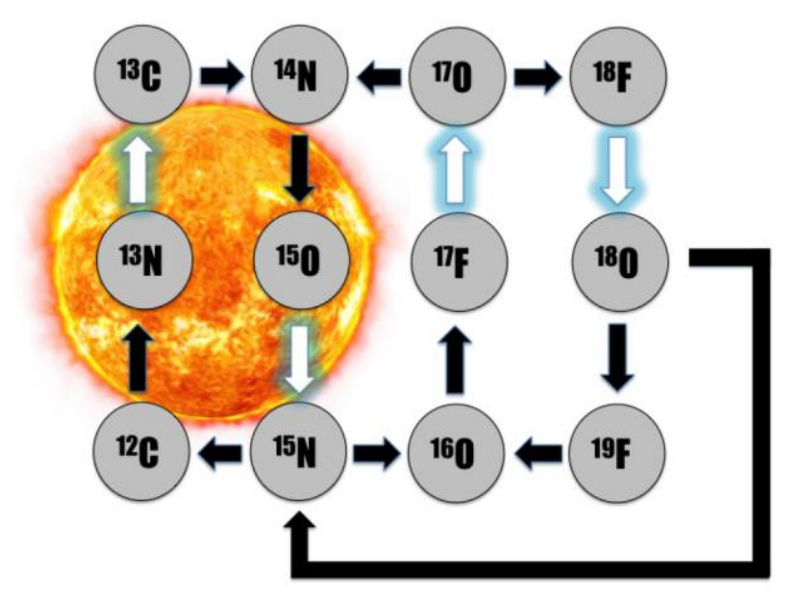

Fig. 2. The cold CNO cycles. The white arrows denote $\beta^{+}$-decays where $v_{e}$ are also released. The leftmost loop is the CNO I cycle relevant for the Sun. Compare with Table 1 and Fig. 1. (See color Figure on the journal website.)

astrophysics. As it is evident from Table 1 and Fig. 1, the corresponding neutrinos have low energies and the only experiment that has a chance to observe them to date is, once again, Borexino. The signal related to the ES reaction is illustrated in Fig. 3. The left panel emphasizes its characteristic shape; the right panel instead shows that the signal of interest occurs in the same region of the one of pep neutrinos, that however are quite well-known being connected to the PP neutrinos and to the solar luminosity. A few remarks are in order:

1. The relative abundances of the species which contribute to the CNO catalysis of hydrogen in helium are precisely predicted by nuclear physics, since they are part of a cycle, whereas the absolute abundance is not and depends upon the model; in the right panel we use the model with high metallicity of Grevesse-Sauval (GS98).

2. Due to the presence of ${ }^{7} \mathrm{Be}$ neutrinos and to the finite resolution of the detector, it is not possible to see the CNO neutrinos for very low energies, $\sim 0.7 \mathrm{MeV}$ in the figure.

3. Borexino contains $278 \mathrm{t}$ of pseudocumene with brute chemical formula $\mathrm{C}_{9} \mathrm{H}_{12}$ and it is working since 10 years.

All in all, the signal is not small and the detection does not seem impossible.
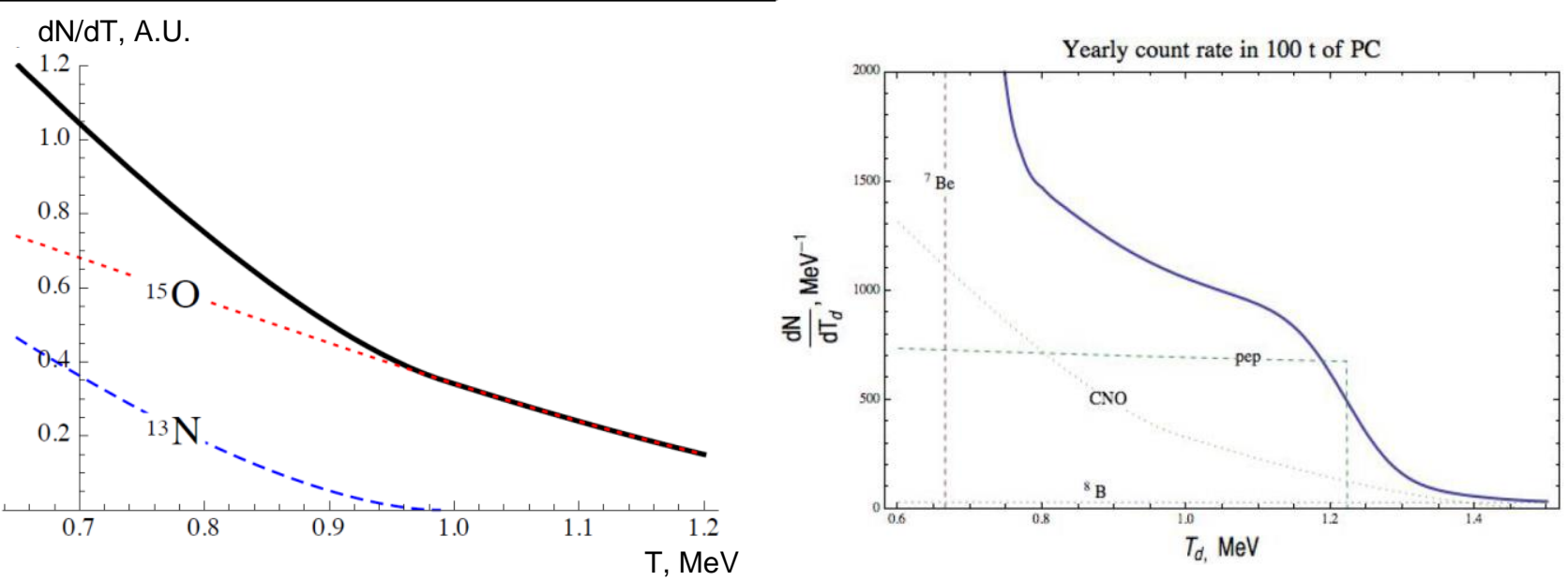

Fig. 3. Spectra due to ES reaction due to CNO neutrinos as a function of the kinetic energy $T_{e}$ of the final state electron. Left panel: the two main components and the sum spectrum, with the characteristic feature at $\sim 1 \mathrm{MeV}$ (the ${ }^{17} \mathrm{~F}$ neutrinos have almost the same shape as the ${ }^{15} \mathrm{O}$ ones but are much less abundant, see Fig. 1). Right panel: yearly count rate in $100 \mathrm{t}$ of pseudocumene (PC), including the effect of energy resolution of $5 \%$ at $T_{e}=1 \mathrm{MeV}$ and showing also the relevant branches of PP neutrinos. (See color Figure on the journal website.)

The real problem is the occurrence of other (background) phenomena due to radioactive contaminants, that could mimic the signal due to CNO neutrinos in which we are interested. At higher energies there is the $\beta^{+}$decay of ${ }^{11} \mathrm{C}$, which can be lowered by more than order of magnitude implementing a three-fold coincidence and that can be further tagged by discriminating the $\beta^{+}$from $\beta^{-}$[12]. At lower energies, which is worse, there is instead a $\beta^{-}$ decay of ${ }^{210} \mathrm{Bi}$, which resembles closely the signal. These considerations are quite evident from Fig. 4 taken from [13]. The last reference is a theoretical investigation of possible strategies aimed at coping with the ${ }^{210} \mathrm{Bi}$ background. The basic point made there is quite simple; the ${ }^{210} \mathrm{Bi}$ is part of a chain of decays, which includes

$$
\begin{aligned}
& { }^{210} \mathrm{~Pb} \stackrel{32.3 y}{\longrightarrow}{ }^{210} \mathrm{Bi}+\bar{v}_{e}+\beta, \\
& { }^{210} \mathrm{Bi} \stackrel{{ }^{2.2 d} \longrightarrow}{\longrightarrow}{ }^{210} \mathrm{Po}+\bar{v}_{e}+\beta, \\
& { }^{210} \mathrm{Po} \longrightarrow{ }_{200 d}{ }^{206} \mathrm{~Pb}+\alpha .
\end{aligned}
$$

This implies a close relationship between the $\beta$ from ${ }^{210} \mathrm{Bi}$ decay and the (intense) signal due to $\alpha$ particle from ${ }^{210}$ Po decay, visible in the leftmost part 
of Fig. 4. This gives a chance to measure the ${ }^{210} \mathrm{Bi}$ contribution, ${ }^{3}$ that can be subtracted from the region of the CNO signal that in this way becomes observable [13].

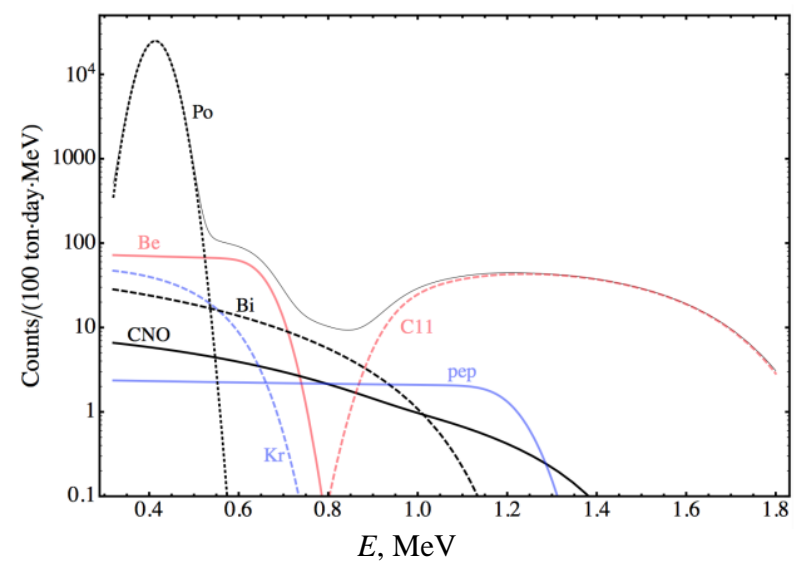

Fig. 4. Spectra of the ES signal due to solar neutrinos (continuous lines) and to background processes (dashed lines). This plot corresponds to the situation in 2011, with 25 count per day/100t of ${ }^{11} \mathrm{C}$ (before three-fold tagging) and with 20 count per day/100t of ${ }^{210} \mathrm{Bi}$. From [13]. (See color Figure on the journal website.)

The reason why since September 2015 Borexino is wrapped in thermal insulation (as can be seen also from Wikipedia [14]) is to keep thermal fluctuations under control, avoiding to reintroduce radioactive contaminants in the detector and attempting the extraction of the CNO signal. In a recent $\mathrm{PhD}$ thesis of a member of Borexino, successfully defended at the GSSI on December 2016, one can read the following words [15]: “... Borexino detector has statistical sensitivity to CNO and pep neutrinos when the dedicated analysis here developed is applied. Central values are $\left(5.2 \pm 1.8_{\text {stat }}\right) \cdot 10^{8} \mathrm{~cm}^{-2} \mathrm{~s}^{-1}$ and $(1.31 \pm$ $\left.\pm 0.35_{\text {stat }}\right) \cdot 10^{9} \mathrm{~cm}^{-2} \mathrm{~s}^{-1} \ldots .$, , that, apart from the misprint $\left(10^{9}\right.$ should be $10^{8}$ as in Figs. 3 or 4$)$, indicates that we are in an exciting moment; developments in the next months are expected.

\section{Particle physics}

The Mikheyev - Smirnov - Wolfenstein theory. The transformation of the electron neutrinos is governed by the interplay between the vacuum oscillation phenomena and the fact that the electron neutrinos propagate differently from the other neutrinos in matter, owing to the fact that they are the only one to undergo charged-current interactions with the elec-

\footnotetext{
${ }^{3}$ E.g., if the detector is stable and no further contaminants are introduced, the average activities $n$ ( $=$ the ratio of the number of nuclei over the lifetime) of the ${ }^{210} \mathrm{Bi}$ and ${ }^{210} \mathrm{Po}$ species are given by $n_{\mathrm{Po}(\mathrm{t})}=n_{\mathrm{Bi}}+\left(n_{\mathrm{Po}(0)}-n_{\mathrm{Bi}}\right) \times$ $\times \exp \left(-t / \tau_{\mathrm{Po}}\right)$; the condition of secular equilibrium obtains for $\tau_{\mathrm{Pb}} \gg t \gg \tau_{\mathrm{Po}}$.
}

trons of the medium as argued by Wolfenstein [16]. The physics is very well-understood and also amenable to a simple description thanks to the analysis of Mikheyev and Smirnov [17]. For instance the neutrinos that reach the detector on day time are in a good approximation (where we neglect the effect of the mixing angle $\theta_{13}$ ) described by the formula

$$
\begin{aligned}
P_{e e}^{\mathrm{day}}\left(E_{v}\right) & \approx \frac{1}{2}\left(1+\cos 2 \theta \cdot \cos 2 \theta_{m}\right) \quad \text { with } \\
\cos 2 \theta_{m} & =\frac{\cos 2 \theta-\varepsilon_{\odot}}{\sqrt{\sin ^{2} 2 \theta+\left(\cos 2 \theta-\varepsilon_{\odot}\right)^{2}}},
\end{aligned}
$$

where $\theta=\theta_{12} \approx 33^{\circ}$ and

$$
\begin{aligned}
\varepsilon_{\odot} & =\frac{\sqrt{2} G_{F} N_{e}^{\odot}}{\Delta m^{2} /\left(2 E_{v}\right)} \approx 1.04\left(\frac{N_{e}^{\odot}}{100 \mathrm{~mol}}\right) \times \\
& \times\left(\frac{7.37 \cdot 10^{-5} \mathrm{eV}^{2}}{\Delta m^{2}}\right)\left(\frac{E_{v}}{5 \mathrm{MeV}}\right)
\end{aligned}
$$

where we have used typical values of the electron neutrino density $N_{e}^{\odot}$ in the region where neutrinos are produced, of the neutrino energy $E_{\mathrm{v}}$ along with the best fit value for $\Delta m^{2}=\Delta m_{12}^{2}$ as indicated by current global analyses of the available data, and mostly by KamLAND reactor antineutrino experiment, based on the assumption that there are 3 light but massive neutrinos.

Interestingly, electron neutrinos that arrive on night time are more abundant, thus the Sun shines brighter in neutrinos during the night! To understand the physics it is sufficient to consider the case when the neutrinos traverse a slab of terrestrial matter of constant density. (An excellent discussion is in [18].) The formula is:

$$
\begin{gathered}
P_{e e}^{\text {night }}\left(E_{v}\right)-P_{e e}^{\text {day }}\left(E_{v}\right)= \\
=\frac{\varepsilon_{\oplus} \cdot \sin ^{2} 2 \theta}{\sin ^{2} 2 \theta+\left(\cos 2 \theta-\varepsilon_{\oplus}\right)^{2}} \times \frac{1 / 2-P_{e e}^{\text {day }}\left(E_{v}\right)}{\cos 2 \theta} .
\end{gathered}
$$

Here $\varepsilon_{\oplus}$ has just the same formal expression as $\varepsilon_{\odot}$ but the electron density in the Earth is of course smaller, $N_{e}^{\odot} » N_{e}^{\oplus}$ and thus $\varepsilon_{\odot} » \varepsilon_{\oplus}$. To date the various observations of solar neutrinos can be accounted for in this simple setup apart from some tension in the overall interpretation, that can be attributed to the following two facts (see [19] for a recent summary and discussion):

1. One would expect that, moving toward the lowest solar neutrino energies currently measured, 
the probability of oscillation should slightly rise, however no sign of turn up is still perceivable.

2. One would not expect that the difference between day and night is very large, however SuperKamiokande measures an effect of electron neutrino regeneration that at central value is twice as expected (with weak significance).

Interestingly, both deviations could be accounted for at the same time, simply assuming that $\Delta m^{2}$ is smaller than stated above, say $4.9 \cdot 10^{-5} \mathrm{eV}^{2}$ as indicated by solar neutrino data alone (dropping Kam-
LAND results from the global fit): see Fig. 5 for an illustration. Another very dramatic and almost certainly premature interpretation is in terms of CPT violation (!!!) namely one could be lead to believe the KamLAND antineutrinos and the solar neutrinos oscillate with different parameters. But plausibly the two results are not due to the same reason and/or are just fluctuations; after all, Borexino verified that the probability of oscillations grows at low energy just as expected.

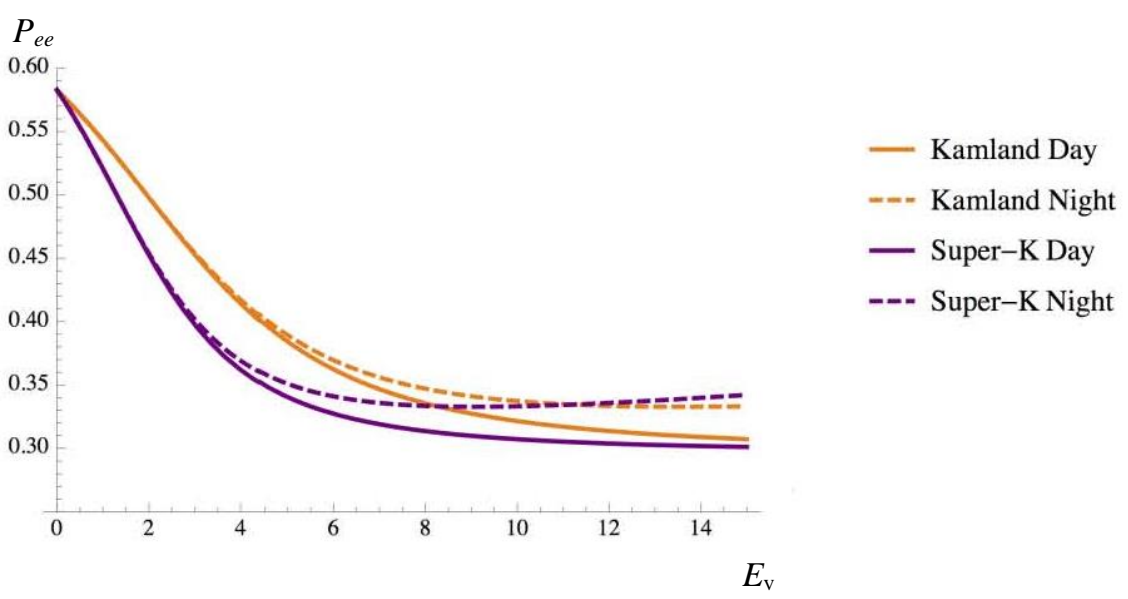

Fig. 5: Illustration of the effect of lowering the value of $\Delta m^{2}$ for the solar neutrino survival probabilities $P_{e e}$ of solar neutrinos. We use the formulae given in the text with the parameters $N_{e}^{\odot}=100 \mathrm{~mol} / \mathrm{cc}$ and $N_{e}^{\oplus}=2 \mathrm{~mol} / \mathrm{cc}$; $\Delta m^{2}($ KamLAND $)=7.37 \cdot 10^{-5} \mathrm{eV}^{2}$ and $\Delta m^{2}($ Super-K $)=4.9 \cdot 10^{-5} \mathrm{eV}^{2}$. (See color Figure on the journal website.)

Speculations. There are many other topics, of great interest for theorists working on particle physics, that can be investigated by means of solar neutrinos. In the following list, we cite some possible manifestations / new particle with a brief comment on the related observable / phenomena:

other light (sterile) neutrinos [shape of the spectrum; neutral current events];

new oscillations on cosmic scales [low energy data];

neutrino decay [flavor structure];

neutrino magnetic moments [solar antineutrinos]; non-standard interactions [new matter-effect]; axions [energy loss];

WIMP in the Sun [solar structure; high-energy neutrinos];

etc.

This list should make evident what is the scientific potential of solar neutrinos for particle physics. Some of them have stronger motivations, some less. Since we do not have evidence of them to date, it seems fair to call them collectively "speculations", until new relevant facts will require a change. We will not enter into a detailed discussion of these topics; we will comment briefly on the first two issues, since they are connected to the existence of other neutrinos and they could affect the phenomenology of neutrino oscillation (just recognized by the Nobel prize committee).
First of all, it is important to stress that, even if it is possible to find several particle physics models that include sterile neutrinos lighter than $\sim 1 \mathrm{eV}$, it is not always clear what are the theoretical motivations for this position. This is quite distinct from the question on whether we have some motivation from experiments, or more precisely, from the interpretation of some experimental fact. To date, one of the few theoretically motivated models is the one based on the concept of mirror symmetry. We describe here the case of exact mirror symmetry, making reference to [20] for a complete discussion. In this model, there is a global $Z_{2}$ symmetry that enforces the existence of mirror bosons and fermions (quarks and leptons) including neutrinos. The mirror particles interact with mirror gauge bosons but do not interact directly with the ordinary ones. In this manner, the mirror baryon may play the role of dark matter. Moreover, mirror neutrinos are exactly degenerate in mass with ordinary neutrinos. In presence of small interactions, e.g., Planck scale suppressed interactions, the ordinary and mirror neutrinos with given mass mix maximally and can give rise to new oscillations at very long scale. This kind of effect can be tested by studying the lowest energy solar neutrinos.

Now, independently on whether we have better or worse theoretical motivations for light sterile 
neutrinos, it is possible to use solar neutrino data to test whether we have any hint of other light neutrinos. A systematic analysis of this type was performed in [21]. Fig. 6, taken from this reference, shows the modification of the pattern of solar neutrino oscillations due to six possible sterile neutri- nos, that were admitted by the data available in 2005. In view of the new data collected since then, it would be useful to repeat this type of analysis. This is interesting per se and potentially relevant in the context of global analyses aiming at finding hints of sterile neutrinos.
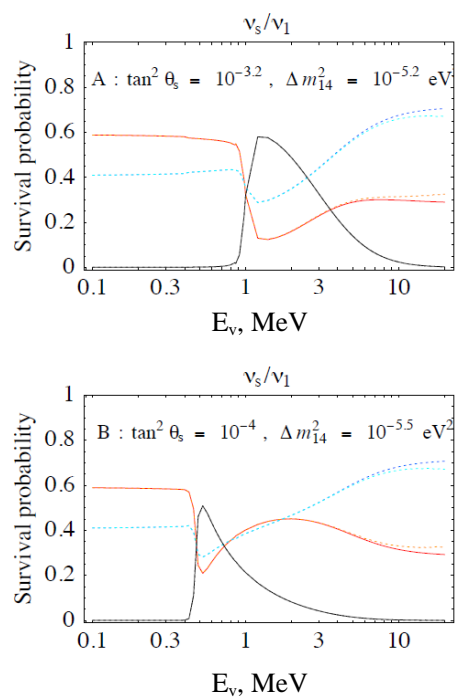
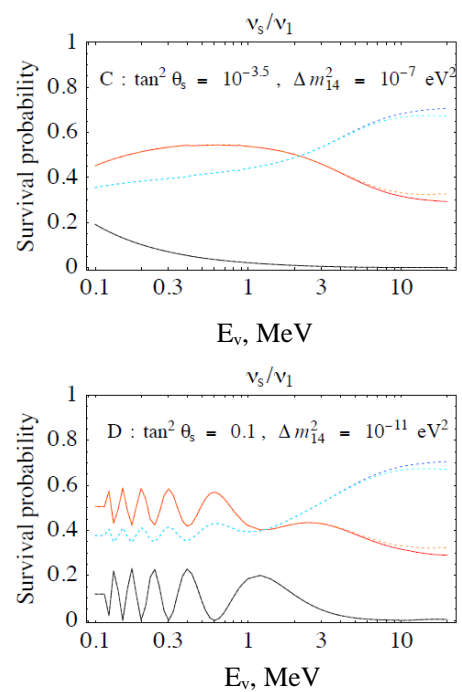
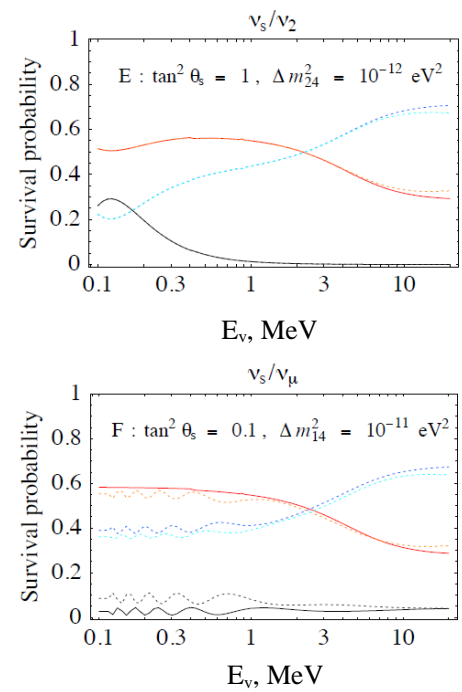

Fig. 6. Sample oscillations patterns of solar neutrinos with light sterile neutrinos. The decreasing red curves give $P\left(v_{e} \rightarrow v_{e}\right)$, the increasing blue curves give $P\left(v_{e} \rightarrow v_{\mu, \tau}\right)$ and the lower black curves give $\mathrm{P}\left(v_{\mathrm{e}} \rightarrow v_{\mathrm{s}}\right)$. The continuous (dotted) curves are the values during day (night). From [21]. (See color Figure on the journal website.)

\section{Remarks}

The study of solar neutrinos is a peculiar branch of neutrino physics, with its own main achievements, burning questions and dynamics of evolution. It is linked with several disciplines such as experimental and theoretical particle physics, nuclear physics and astrophysics. These links contribute greatly to maintain solar neutrino physics lively. As we have argued, solar neutrino physics is today in a healthy state, just as neutrino physics in general see [22] for a wider discussion.

Coming to specific considerations, we have shown that a definitive understanding of how the Sun functions was obtained only 3 years ago by Borexino experiment at Gran Sasso laboratory. We mean here "understanding" in a Galilean sense, namely, we have been able to test the hypotheses (i.e., the theoretical expectations) by means of experiments and observations. The same team is progressing with more goals ahead: We are on the verge of learning on CNO neutrinos. A lot of physics issues can be usefully investigated and surprises may still occur. It should be not forgotten that such topics are of great interest for a very wide audience - it is easy to explain to a wide public that we have understood how the Sun works. Moreover, the Nobel prize in physics, assigned for the beginning of neutrino astronomy in 2002, witnesses that similar activities are also considered worth of reward by the scientific community.

The last remark is sociological in nature: whether we want it or not, neutrino astronomy is largely in the hands of particle physicists. This is true also, to a good extent, for the more specific branch of solar neutrino studies. Such a circumstance is not necessarily good or bad, however, in view of the healthy state of the field, it can be considered fortunate. It is also a reason of special responsibility: in particular, theorists working in particle physics are supposed to pay attention to the features of the field. In this manner, they will play an even more important role and will take great advantages from solar neutrinos.

I thank G. Barenboim, G. G. Raffelt for the invitation at CERN; A. Strumia, F. L. Villante for collaboration on solar neutrinos; G. Bellini, P. Sapienza for precious discussions; A. Smirnov and L. Lavoura for providing useful feedback.

\section{REFERENCES}

1. Bahcall J.N. Neutrino Astrophysics. - Cambridge University Press, 1989. - 567 p.

2. Raffelt G.G. Stars as Laboratories for Fundamental Physics: The Astrophysics of Neutrinos, Axions, and Other Weakly Interacting Particles. - USA, Chicago:
Univ. Pr., 1996. - 664 p.

3. Underground nuclear astrophysics and solar neutrinos: Impact on astrophysics, solar and neutrino physics / Eds. G. Bellini, C. Broggini, A. Guglielmetti. - Access mode: 
https://link.springer.com/journal/10050/topicalColle ction/AC_b8906a62dc1e484620b205ce85f25e74

4. Winter W.T., Freedman S.J., Rehm K.E., Schiffer J.P. The B-8 neutrino spectrum // Phys. Rev. C . 2006. - Vol. 73. - P. 025503.

5. Adelberger E.G., Austin S.M., Bahcall J.N. et al. Solar fusion cross sections // Rev. Mod. Phys. 1998. - Vol. 70. - P. 1265.

6. Chen H.H. Direct Approach to Resolve the Solar Neutrino Problem // Phys. Rev. Lett. - 1985. Vol. 55. - P. 1534.

7. Bahcall J.N., Serenelli A.M., Basu S. New solar opacities, abundances, helioseismology, and neutrino fluxes // Astrophys. J. - 2005. - Vol. 621. - P. L85.

8. Vinyoles N., Serenelli A.M., Villante F.L. et al. A new Generation of Standard Solar Models // Astrophys. J. - 2017. - Vol. 835, No. 2. - P. 202. [arXiv:1611.09867 [astro-ph.SR]].

9. D'Angelo D., Bellini G., Benziger J. et al. [Borexino Collaboration]. Recent Borexino results and prospects for the near future // EPJ Web Conf. 2016. - Vol. 126. - P. 02008.

10. Raghavan R.S., Pakvasa S., Brown B.A. New Tools for Solving the Solar Neutrino Problem // Phys. Rev. Lett. - 1986. - Vol. 57. - P. 1801.

11. Bellini G., Benziger J., Bick D.G. et al. [Borexino Collaboration]. Neutrinos from the primary protonproton fusion process in the Sun // Nature. - 2014. Vol. 512, No. 7515. - P. 383.

12. Bellini G., Benziger J., Bick D.G. et al. [Borexino Collaboration]. Final results of Borexino Phase-I on low energy solar neutrino spectroscopy // Phys. Rev. D. - 2014. - Vol. 89. - P. 112007.
13. Villante F.L., Ianni A., Lombardi F. et al. A Step toward CNO solar neutrinos detection in liquid scintillators // Phys. Lett. B. - 2011. - Vol. 701. P. 336.

14. Access mode: https://en.wikipedia.org/wiki/Borexino

15. Drachnev I. New Spectral Analysis of Solar ${ }^{8} \mathrm{~B}$ Neutrino with the Borexino Detector: PhD Thesis submitted on October 28, 2016 / Gran Sasso Science Institute, Astroparticle Physics Doctoral Programme.

16. Wolfenstein L. Neutrino Oscillations in Matter // Phys. Rev. D. - 1978. - Vol. 17. - P. 2369.

17. Mikheyev S.P., Smirnov A.Y. Resonance Amplification of Oscillations in Matter and Spectroscopy of Solar Neutrinos, Sov. J. Nucl. Phys. - 1985. Vol. 42. - P. 913; Yad. Fiz. - 1985. - Vol. 42. P. 1441; Nuovo Cim. C. - 1986. - Vol. 9. - P. 17.

18. Lisi E., Montanino D. Earth regeneration effect in solar neutrino oscillations: An Analytic approach // Phys. Rev. D. - 1997. - Vol. 56. - P. 1792.

19. Abe K., Haga Y., Hayato Y. et al. [Super-Kamiokande Collaboration]. Solar Neutrino Measurements in Super-Kamiokande-IV // Phys. Rev. D. - 2016. Vol. 94. - P. 052010.

20. Berezinsky V., Narayan M., Vissani F. Mirror model for sterile neutrinos // Nucl. Phys. B. - 2003. Vol. 658. - P. 254.

21. Cirelli M., Marandella G., Strumia A., Vissani F. Probing oscillations into sterile neutrinos with cosmology, astrophysics and experiments // Nucl. Phys. B. - 2005. - Vol. 708. - P. 215.

22. Vissani $F$. La Thuile 2014: Theoretical premises to neutrino round table // Nuovo Cim. C. - 2014. Vol. 037, No. 6. - P. 66.

\section{Ф. Віссані \\ Національний інститут ядерної фізики, Національна лабораторія Гран Сассо та Науковий інститут Гран Сассо, Аквіла, Італія}

\section{ФІЗИКА СОНЯЧНИХ НЕЙТРИНО НА ПОЧАТКУ 2017 Р.}

Представлено огляд сучасних «гарячих» питань в області сонячних нейтрино. Огляд базується на доповіді на конференції «Нейтрино: пошуки нової фізичної шкали», що відбулася в ЦЕРНі в березні 2017 р., де організатори довірили мені обговорення провокаційного питання «чи закінчилась фізика сонячних нейтрино». Замість того, щоб дати прямолінійну (негативну) відповідь, зважаючи на аудиторію, яка складалася головним чином із колег, які працюють у галузі теоретичної фізики частинок, я вважав більш корисним дати сучасний опис діяльності фізиків, які працюють над сонячними нейтрино, дозволяючи слухачам сформувати власну думку.

Ключові слова: сонячні нейтрино, нейтринні осциляції, ядерна астрофізика, рр цикл, СNO цикл.

\section{Ф. Виссани}

Национальный институт ядерной физики, Нацииоальная лаборатория Гран Сассо и Научный институт Гран Сассо, Аквила, Италия

\section{ФИЗИКА СОЛНЕЧНЫХ НЕЙТРИНО В НАЧАЛЕ 2017 Г.}

Представлен обзор современных «горячих» вопросов в области солнечных нейтрино. Обзор основан на докладе на конференции «Нейтрино: поиски новой физической шкалы», которая состоялась в ЦЕРНе в марте 2017 г., где организаторы доверили мне обсуждение провокационного вопроса «закончилась ли физика солнечных нейтрино». Вместо того, чтобы дать прямолинейный (негативный) ответ, принимая во внимание аудиторию, которая состояла в основном из коллег, работающих в области теоретической физики частиц, я счел более полезным дать современный обзор деятельности физиков, работающих над солнечными нейтрино, позволяя слушателям сформировать собственное мнение.

Ключевые слова: солнечные нейтрино, нейтринные осцилляции, ядерная астрофизика, рр цикл, СNO цикл. 